\title{
Lipid nanoparticles that deliver IL-12 messenger RNA suppress tumorigenesis in MYC oncogene-driven hepatocellular carcinoma
}

\author{
Ian Lai ${ }^{1+}$, Srividya Swaminathan ${ }^{1 \dagger}$, Virginie Baylot ${ }^{1}$, Adriane Mosley $^{1}$, Renumathy Dhanasekaran ${ }^{2}$, Meital Gabay ${ }^{1}$ \\ and Dean W. Felsher ${ }^{1 *}$
}

\begin{abstract}
Interleukin-12 (IL-12) is a promising candidate for cancer immunotherapy because of its ability to activate a number of host immune subsets that recognize and destroy cancer cells. We found that human hepatocellular carcinoma (HCC) patients with higher than median levels of IL-12 have significantly favorable clinical outcomes. Here, we report that a messenger RNA (mRNA) lipid nanoparticle delivering IL-12 (IL-12-LNP) slows down the progression of MYC oncogene-driven HCC. IL-12-LNP was well distributed within the HCC tumor and was not associated with significant animal toxicity. Treatment with IL-12-LNP significantly reduced liver tumor burden measured by dynamic magnetic resonance imaging (MRI), and increased survival of MYC-induced HCC transgenic mice in comparison to control mice. Importantly, IL-12-LNP exhibited no effect on transgenic MYC levels confirming that its therapeutic efficacy was not related to the downregulation of a driver oncogene. IL-12-LNP elicited marked infiltration of activated $\mathrm{CD}_{4} 4^{+} \mathrm{CD}^{+} \mathrm{CD}^{+} \mathrm{T}$ helper cells into the tumor, and increased the production of Interferon $\gamma$ (IFNY). Collectively, our findings suggest that IL-12-LNP administration may be an effective immunotherapy against HCC.
\end{abstract}

Keywords: HCC, IL-12, Immunotherapy

\section{Introduction}

Cytokines are crucial signaling mediators that alert the immune system to the presence of foreign antigens. Cytokine treatment may also be an effective immune therapy for the treatment of cancer. Interleukin-12 (IL-12) has anti-tumor activity in a variety of preclinical models [1-6]. The anti-tumor activity of IL-12 can be attributed to its ability to bridge innate and adaptive immune surveillance mechanisms, thereby creating a long-lasting immune response against cancers [7].

IL-12 was originally identified as "natural killer (NK) cell stimulatory factor" [8], but has subsequently been shown to exert effects on other immune compartments such as NK T cells, T-lymphocytes [9], cells of myeloid

\footnotetext{
* Correspondence: dfelsher@stanford.edu

${ }^{\dagger}$ Ian Lai and Srividya Swaminathan contributed equally to this work.

${ }^{1}$ Division of Medical Oncology, Departments of Medicine and Pathology,

Stanford University, CA, Stanford, USA

Full list of author information is available at the end of the article
}

origin [10], and tonsillar B cells [11]. Despite its pleiotropic effects on multiple immune compartments, IL-12 is particularly important during $\mathrm{T}$ cell activation initiated by $\mathrm{T}$ cell receptor (TCR) signaling $[12,13]$. TCR activation promotes sensing of IL-12 by the induction of IL-12 receptors (IL-12R), and subsequently leads to secretion of Interferon- $\gamma$ (IFN $\gamma$ ) by the activated T cells [14]. IFN $\gamma$ can kill tumor cells directly or by recruiting and activating key immune subsets such as macrophages and NK cells that recognize tumor cells [15]. Thus, IL-12 is a potent anti-cancer agent directly and indirectly through the recruitment and activation of other immune effectors, and secretion of cytokines.

Administration of IL-12 has been previously demonstrated to have therapeutic benefits in multiple preclinical models of cancer, such as breast [16], liver [17, 18], and colon [19]. However, the lack of intravenous approaches to robustly deliver IL-12 to the tumor and avoid cytotoxicity of the surrounding normal tissues has 
slowed the development of IL-12 as a modality to treat human cancers [20].

Amongst solid tumors, treatment of hepatocellular carcinoma (HCC) remains a significant clinical challenge [21-24]. Hence, in the current study, we examined the efficacy of a novel mRNA lipid nanoparticle based approach for the in situ delivery and production of IL-12 (IL-12-LNP) in suppressing tumor progression in a primary transgenic mouse model of refractory MYC-driven hepatocellular carcinoma (HCC, [25]). Overexpression of the MYC oncogene is a common feature of aggressive HCCs [26-29]; making our model particularly relevant for pre-clinical studies involving novel therapeutic agents, such as IL-12-LNP.

While molecular targeting of MYC would represent the most ideal treatment strategy for combating MYC-driven HCCs [30-33], MYC inhibitors that do not exert toxic effects on normal tissues are yet to be identified. By selecting our model of MYC-driven HCC, we hoped to identify if IL-12-LNP can be an effective non-toxic alternative to MYC inhibition for treating aggressive HCCs. We demonstrate that IL-12-LNP is well distributed to the liver tumor and surrounding normal liver tissue, not associated with animal and liver toxicity, suppresses tumor growth, and increases survival of our transgenic mice predisposed to developing MYC-driven HCC.

\section{Methods}

\section{Human HCC patient data mining and analysis}

Gene expression data were analyzed from a previously published retrospective study conducted on 387 paraffin-embedded liver tissues obtained from HCC patients (Gene Expression Omnibus (GEO) Accession Number GSE10143) [34]. Normalized mRNA expression data and clinical data (survival times and outcomes) of patients $(n=387)$ were obtained from PRECOG (Prediction of Clinical Outcomes from Genomic Profiles, https://precog.stanford.edu/index.php). Patients $(n=144)$ for whom survival data was available were separated into two groups based on the median levels of IL12A mRNA, as IL12A ${ }^{\text {High }}$ and IL12A ${ }^{\text {Low }}$. Kaplan-Meier Survival Analysis was then used to compare the overall survival (OS) probabilities between the two groups to determine if IL12A mRNA levels in liver tissue can serve as an independent predictor of clinical outcome in HCC patients.

\section{HCC transgenic mice}

All animals were housed in a pathogen-free environment at Stanford University and all procedures were performed in accordance with Stanford's Administrative Panel on Laboratory Animal Care (APLAC) protocols. LAP-tTA/tet-O-hMYC transgenic lines, as previously described [25] (Fig. 1b, Model), were administered weekly doses of $0.1 \mathrm{mg} / \mathrm{mL}$ doxycycline (Sigma) in drinking water during mating (in utero) up until 4 weeks of age. Mice were screened for tumors under $50 \mathrm{~mm}^{3}$, developing at approximately $2-3$ months of age, with euthanasia occurring following completion of treatment or when tumor burden exceeded $5,000 \mathrm{~mm}^{3}$.

\section{Magnetic Resonance Imaging (MRI)}

MRI was performed weekly using a $7 \mathrm{~T}$ small animal MRI (Brucker) at the Stanford Small Animal Imaging Facility as previously described [35]. Briefly, animals were anesthetized with $1-3 \%$ isofluorane and fed into the MRI scanner containing a $40 \mathrm{~mm}$ Varian Millipede RF coil (ExtendMR LLC). Tumor volumes were quantified from acquired DICOM images using Osirix image processing software (Osirix).

\section{Lipid Nanoparticle (LNP) administration}

Modified mRNA LNPs containing IL-12 or a control oligonucleotide containing no start codon (NST-LNP) were synthesized and provided by Onkaido (Moderna Therapeutics). Upon tumor detection, mice were administered weekly intravenous doses of the NST-LNP $(0.025 \mathrm{mg} / \mathrm{kg})$ or IL-12-LNP $(0.025 \mathrm{mg} / \mathrm{kg})$, for 3 weeks (20 mice per group) or up to 9 weeks for the determination of a survival curve (14-15 mice per group).

\section{Immunohistochemistry}

Tissues were fixed in 10\% paraformaldehyde and embedded in paraffin for sectioning. Sections were deparaffinized and stained with c-MYC (1:150, Clone \#EP121, monoclonal, Epitomics), CD4 (1:100, Clone \#EPR19514, monoclonal, Abcam), CD3 (1:100, polyclonal, Abcam), and CD44 (1:2000, polyclonal, Abcam) overnight, and incubated with biotinylated anti-mouse IgG for $30 \mathrm{~min}$ at room temperature (1:300 Vectastain $A B C$ kit, Vector Labs). Sections were developed using 3,3' - Diaminobenzidine (DAB), counterstained with hematoxylin, and mounted with permount. Stained sections were scanned and imaged with a Digital Pathology Slide Scanner (Philips). Representative tumor tissue sections were quantified from acquired images using ImageJ software (NIH).

\section{Quantitative RT-PCR}

Quantitative real-time PCR carried out with the SYBRGreenER mix from Invitrogen according to standard PCR conditions and an ABI7900HT real-time PCR system (Applied Biosystems). Primers for quantitative RT-PCR are listed below:

mGOT1_F 5'-AGAGAAAGATGCGTGGGCTA-3'. mGOT1_R 5'-TGGACCAGGTGATTCGTACA-3'. mGPT_F 5'-AAGGCTAAACTCACGGAGCA-3'. mGPT_R 5'-CTCTTCCAGGAGGCACAGAC-3'. mALPL_F 5'-GCTGATCATTCCCACGTTTT-3'. 


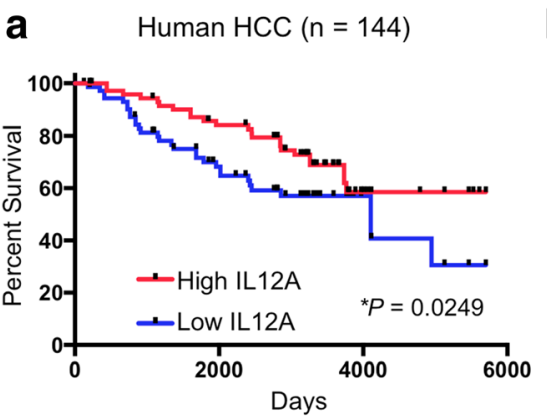
b TRE-MYC LAP tTA (HCC-bearing mice)
Overt HCC, No Doxycycline, MYCON
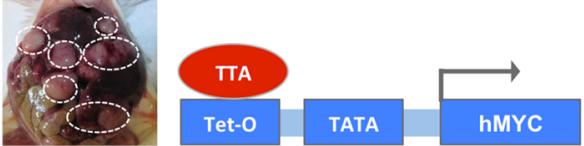

Regressed HCC, Doxycycline (DOX), MYC ${ }^{\text {OFF }}$

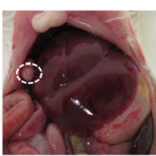

DOX

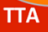

Tet-o TATA hMYc

C

Distribution of GFP-LNP (HCC-bearing mice)

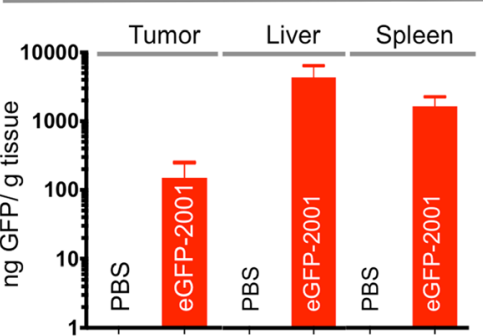

e

Liver Transaminase mRNA (HCC-bearing mice)

d

Normal liver tissue in HCC-bearing mice (H\&E)
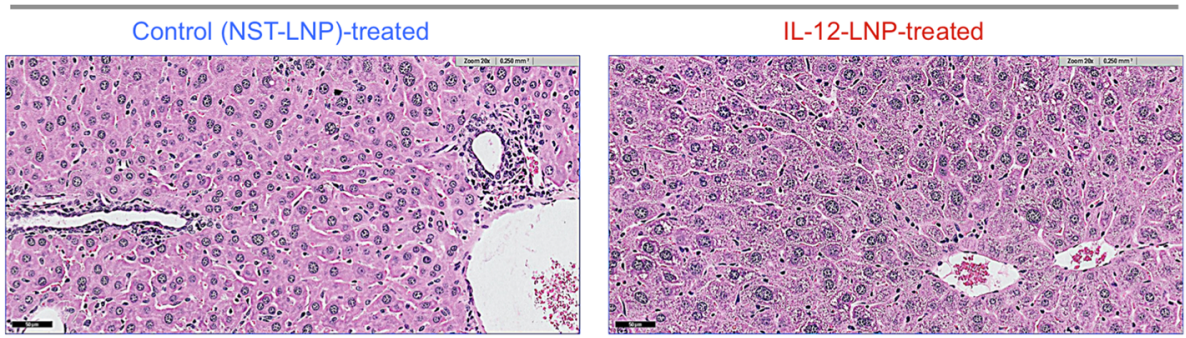

Fig. $1 \mathrm{IL}-12-L N P$ as an effective and non-toxic treatment for hepatocellular carcinoma. a Kaplan-Meier survival analysis comparing overall survival (OS) probabilities of HCC patients $(n=144)$ divided into two groups (High IL-12 and Low IL-12) based on their median expression of IL-12. P-value was calculated using the log-rank test. $\mathbf{b}$ Morphology of livers (left) and diagrammatic representation (right) of the transgenic mouse model of MYC-driven HCC used in the study, before and after MYC inactivation. White dotted circles represent examples of tumor nodules in the liver. c Biodelivery of IL-12 oligonucleotide therapy (IL-12-LNP) and corresponding controls (NST-LNP) designed by Onkaido, in HCC (tumor, left), whole liver (middle), and spleen (right). d Hematoxylin \& Eosin (H\&E) analysis of normal liver tissue surrounding tumors isolated from control (NST-LNP, $n=7)$ and IL-12 treated (IL-12-LNP, $n=7)$ HCC-bearing mice. One representative image is shown from each group of mice. Scale bars $=50 \mu m$. $\mathbf{e}$ Quantitative real time PCR comparing mRNA levels of liver transaminases between NST-LNP $(n=7)$ and IL-12-LNP $(n=7)$ treated HCC-bearing mice. Each dot represents a single mouse and is the average of three technical replicates. Data are represented as median \pm interquartile range. $P$-values were calculated using Student's t-test. $P$ values: ns $=$ not significant, ${ }^{*} p<0.05,{ }^{* *} p<0.01,{ }^{* * *} p<0.001,{ }^{* * * *} p<0.0001$

mALPL_R 5'-CTGGGCCTGGTAGTTGTTGT-3'. mIFNG_F 5'-ACTGGCAAAAGGATGGTGAC-3'. mIFNG_R 5'-TGAGCTCATTGAATGCTTGG-3'.

\section{Statistical analysis}

Results (Mean \pm SEM) were analyzed for statistical significance by Student's t-test and one-way ANOVA, using Prism (Graphpad Software, Inc.). The Kaplan-Meier estimate was used for survival analysis. Log-rank test was used to calculate statistical significance for all survival studies. The threshold for statistical significance was set at $P<0.05 . P$ values: $n s=$ not significant, ${ }^{*} p<0.05$, *\%* $p<0.001$, ${ }^{* * * * *} p<0.0001$.

\section{Results}

IL-12 expression predicts survival of humans with HCC Gene expression profiles of surrounding normal liver tissues have been previously demonstrated to predict the 
prognosis of patients with hepatocellular carcinoma (HCC, GSE10143) [34]. We examined if the expression of IL-12 mRNA in the surrounding normal liver tissue of human HCC patients can predict clinical outcome. A cohort of 144 HCC patients (GSE10143) were divided into two groups based on median expression of IL-12 mRNA levels [34] (Fig. 1a). Patients with higher than median IL-12 mRNA levels in normal liver tissue surrounding the HCC had a significantly prolonged overall survival (OS) than patients with lower than median IL-12 levels (Fig. 1a).

Since IL-12 is a favorable prognostic factor for HCC, we hypothesized that HCC may respond to IL-12-based therapies. We evaluated the efficacy of a novel IL-12 mRNA therapy in our primary tetracycline (tet)-inducible transgenic mouse model of MYC oncogene-driven HCC [25] (Fig. 1b). In this transgenic model, full-blown HCC tumors completely regress only upon MYC inactivation (Fig. 1b). We investigated whether IL-12 mRNA-based therapy can delay MYC-driven HCC progression, and therefore serve an alternative to MYC inhibition for treating HCCs.

\section{A non-toxic IL-12 mRNA-based immunotherapy for HCC}

Nucleic acids, mRNA in particular, are beginning to be widely employed in cancer immunotherapy [36-40]. Here, we use a modified IL-12 mRNA encapsulated within a lipid nanoparticle (LNP), referred as "IL-12-LNP". The IL-12 mRNA was engineered to have the desired sequence and modifications for producing a functionally active IL-12 protein. Once within the organism, the LNP releases the IL-12 mRNA into cells where it is decoded by the ribosomal machinery into IL-12 protein. A control oligonucleotide with no start codon (NST) encapsulated in LNPs (referred here as "NST-LNP" controls) was used as a vehicle control. Both the IL-12-LNP and NST-LNP were delivered intravenously.

First we measured the biodistribution of intravenously administered LNPs carrying a GFP tagged NST mRNA cargo in our transgenic mouse model of MYC-driven HCC. NST-GFP-LNP was efficiently delivered to the tumor (HCC, Fig. 1c, left), non-malignant regions of the liver (Fig. 1c, middle) and spleen (Fig. 1c, right) of the mice, suggesting its superiority over existing intratumoral methods of IL-12 delivery [20].

Next, we evaluated whether IL-12-LNP treatment induces animal toxicity. The administration of IL-12-LNP did not significantly alter the body weights of healthy mice as compared to NST-LNP-treated controls (Additional file 1a). We found that normal tissue surrounding liver tumors in HCC-bearing mice show no evidence of increased hepatitis post IL-12-LNP treatment in comparison to NST-LNPtreated controls (Fig. 1d). To definitively rule out liver toxicity because of IL-12-LNP treatment, we also compared the mRNA levels of common liver transaminases (Glutamic-Oxaloacetic Transaminase 1, GOT1; Alkaline Phosphatase, ALPL; Glutamic Pyruvic Transaminase, GPT) in normal liver tissue between NST-LNP and IL-12-LNP treated HCC-bearing mice (Fig. 1e). We observed that IL-12-LNP treatment does not significantly stimulate the production of liver transaminases (Fig. 1e). Based on these findings, we conclude that IL-12-LNP therapy does not induce liver toxicity.

\section{Short-term administration of IL-12-LNP significantly reduces $\mathrm{HCC}$ tumor burden}

The effects of short-term (3 week) treatment with IL-12-LNP $(n=20)$ versus NST-LNP controls $(n=20)$ on tumor progression in a transgenic mouse model of MYC-driven HCC [25] were measured by magnetic resonance imaging (MRI). MRI is a sensitive methodology to robustly visualize the dynamic process of hepatocellular carcinogenesis over a given period of time. The short-term IL-12-LNP administration significantly reduced liver tumor burden and delayed tumor progression when compared to NST-LNP-treated controls (Fig. 2a-b).

HCC transgenic mice in both groups (control and IL-12-treated) had comparable tumor burdens (0$50 \mathrm{~mm}^{3}$ ) at the start of treatment (week 0, Fig. 2c). At week 3 , we observed a significant reduction in the absolute tumor burden in the IL-12-treatment group (Fig. 2d). HCC progression was quantified as a ratio of the change in tumor burden at week 3 with respect to week 0 for individual mice in each group (Fig. 2e). HCC progression was significantly delayed in IL-12-treatment cohort as compared to the NST-LNP-treated control group (Fig. 2e). Concordant with the reduced tumor growth measured by MRI, there was a reduction in the gross number of tumor nodules in liver, in mice sacrificed three weeks after IL-12-LNP administration (Fig. 2f). Hence, IL-12-LNP treatment significantly reduces $\mathrm{HCC}$ tumor growth and delays tumor progression as demonstrated by dynamic MRI.

\section{Treatment with IL-12-LNP confers a significant survival advantage in transgenic mice}

The effects of long-term administration of IL-12-LNP were examined in MYC-driven $\mathrm{HCC}$ transgenic mice. Two cohorts of HCC-bearing mice $\left(0-50 \mathrm{~mm}^{3}\right)$ were each given IL-12-LNP $(n=14)$ or control (NST-LNP, $n$ $=15$ ) until the mice were moribund with tumor burden (Fig. 3). IL-12-LNP administration significantly delayed tumor progression and prolonged overall survival of mice in comparison to NST-LNP controls (Fig. 3a). The long-term administration of IL-12-LNP reduced the 

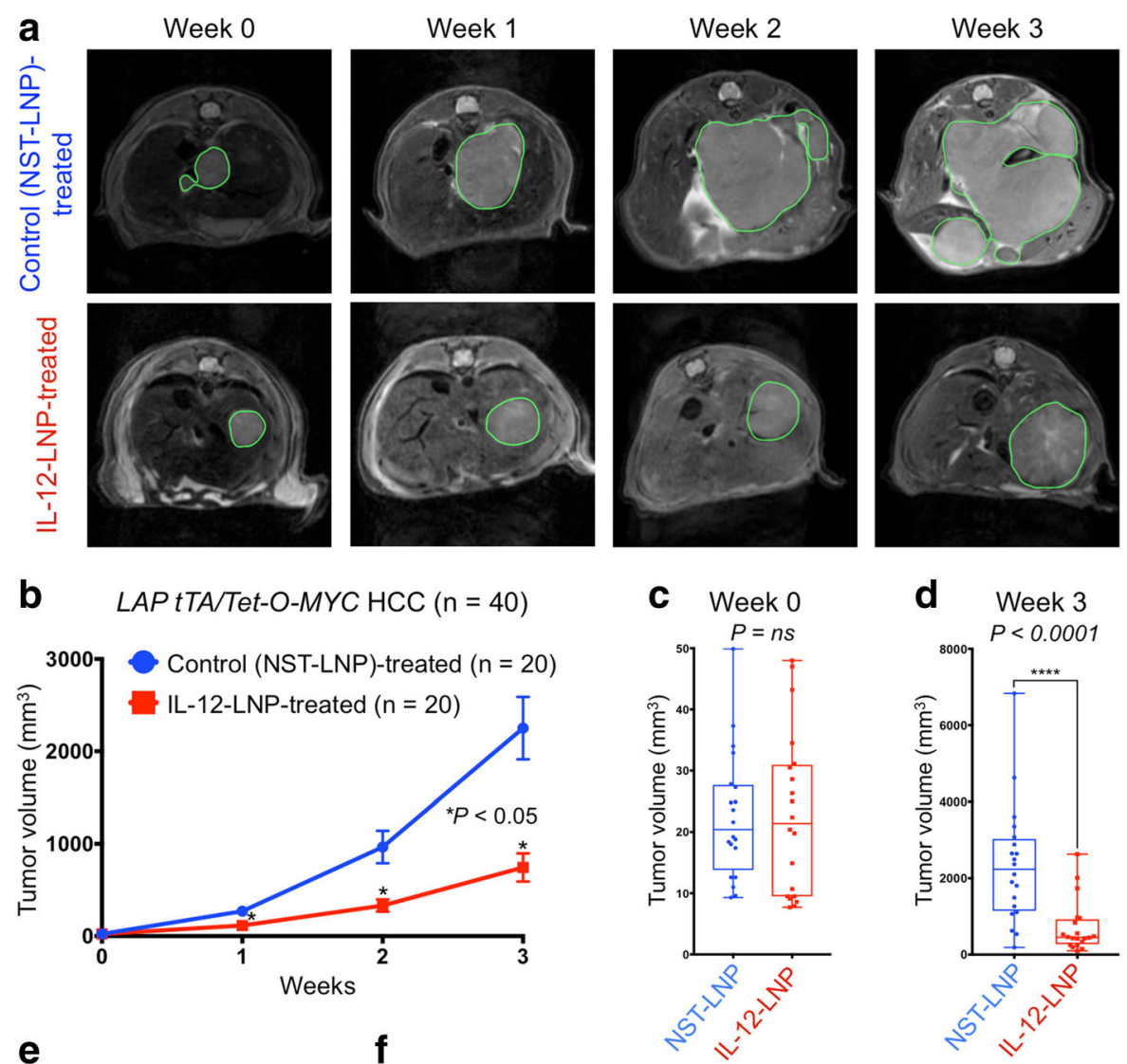

e

HCC progression

(Tumor volume week $3 /$

Tumor volume week 0)
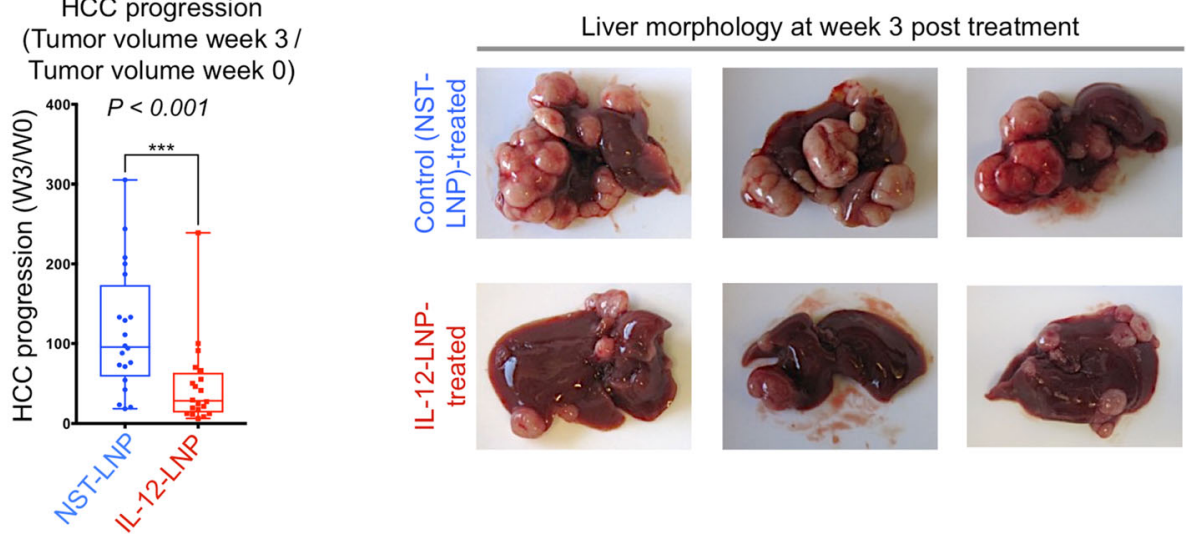

Fig. 2 Short-term treatment with IL-12-LNP significantly slows down HCC progression. a Serial magnetic resonance imaging (MRI) of primary transgenic mice bearing MYC-driven HCC that were either administered control NST-LNP $(n=20)$ or IL-12-LNP $(n=20)$ intravenously at weeks 0, 1 , 2 and 3 post treatment. Representative images of one mouse in each group are shown for the indicated time points. $\mathbf{b}$ Comparison of HCC progression (tumor burden) as computed by MRI after treatment with control (NST-LNP, $n=20$ ) or IL-12 (IL-12-LNP, $n=20)$ oligonucleotides for three weeks. Tumor burden for each time point represented as mean \pm SEM. c Box plots depicting absolute HCC burden in individual mice before starting treatment with IL-12-LNP $(n=20)$ or NST-LNP $(n=20)$ controls (week 0$)$, as computed by MRI. d Box plots depicting absolute HCC burden in individual mice at the end of short-term treatment with IL-12-LNP $(n=20)$ or NST-LNP $(n=20)$ controls (week 3), as computed by MRI. e Box plots depicting fold change in HCC burden of individual mice at week 3 with respect to initial tumor burden before beginning treatment (week 0) between NST-LNP and IL-12-LNP treated groups. P-values were calculated using Student's t-test. P values: ns $=$ not significant, ${ }^{*} \mathrm{p}<0.05$, ${ }^{* * *} p<0.001$, *** $p<0.0001$. f Morphology of livers isolated from control (NST-LNP, $\mathrm{n}=20$ ) and IL-12-treated (IL-12-LNP, $\left.\mathrm{n}=20\right) \mathrm{HCC}$ mice at week 3 post treatment. Three representative images are shown from each group 
a

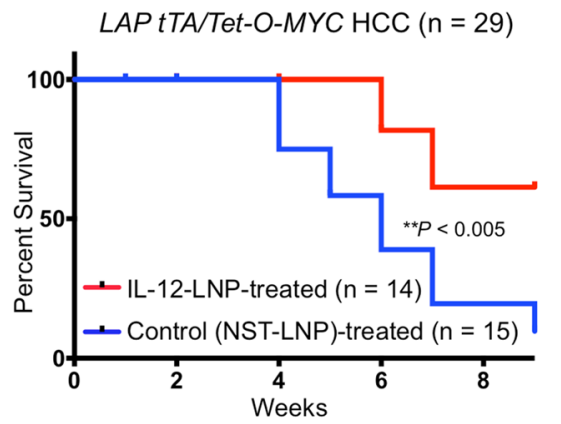

b

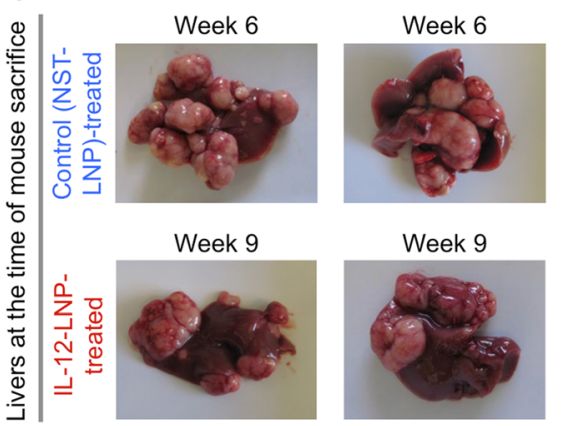

C Liver tumor in HCC-bearing mice (H\&E)
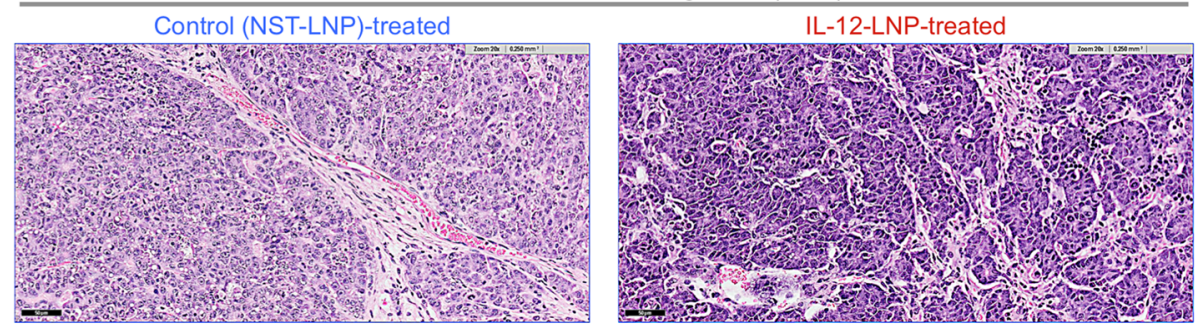

d

Liver tumor in HCC-bearing mice (MYC)
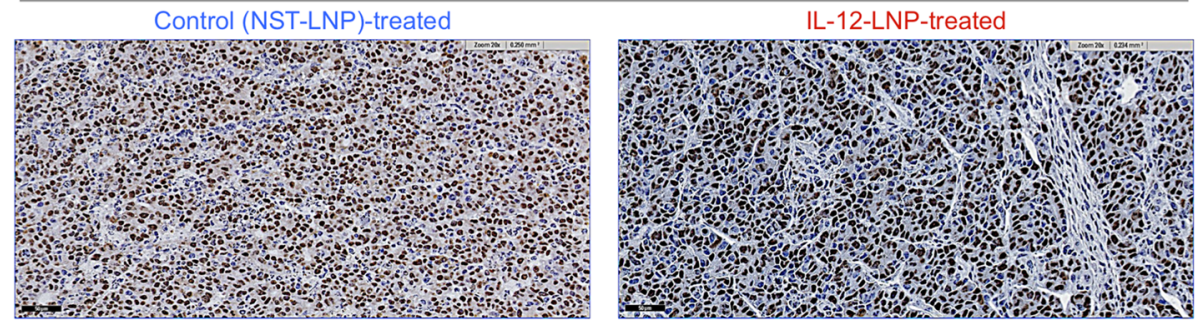

e

Normal liver tissue in HCC-bearing mice (MYC)
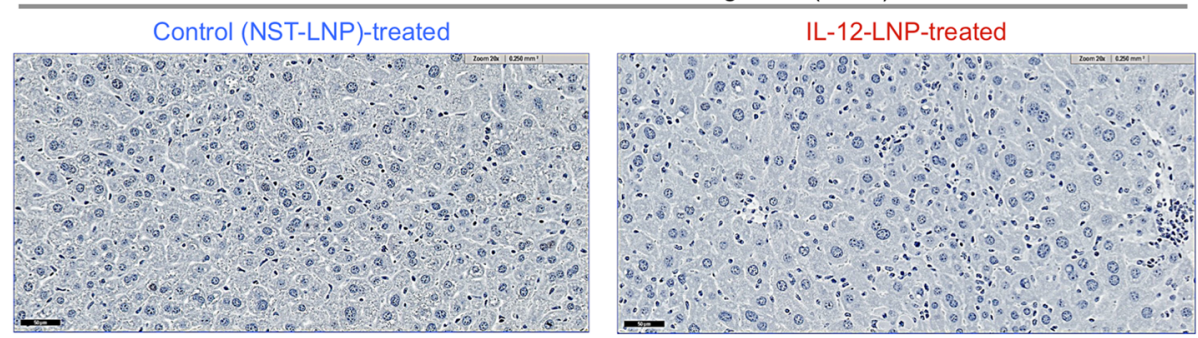

Fig. 3 Treatment with IL-12-LNP confers a significant survival advantage on HCC-bearing mice independently of MYC. a Kaplan-Meier survival analysis comparing overall survival (OS) between control (NST-LNP, $n=15)$, and IL-12-treated (IL-12-LNP, $n=14)$ MYC-driven HCC mice. P-values were calculated using log-rank test. P values: ${ }^{* *} p<0.01$. b Morphology of livers isolated from control (NST-LNP, $\left.\mathrm{n}=15\right)$ and IL-12-treated (IL-12$L N P, n=14)$ HCC mice at the end of treatment schedule. Two representative images are shown from each group of mice. $\mathbf{c}$ Hematoxylin \& Eosin (H\&E) analysis of liver tumors isolated from control (NST-LNP, $n=7)$ and IL-12 treated (IL-12-LNP, $n=7)$ HCC-bearing mice at the end of treatment. d-e Immunohistochemistry for transgenic hMYC protein in liver tumor (d), and surrounding normal liver (e) tissues isolated from control (NST-LNP, $n=7$ ) and IL-12 treated (IL-12-LNP, $n=7$ ) MYC-driven HCC mice. One representative image is shown from each group of mice. Scale bars $=50 \mu \mathrm{m}$

disease burden as measured by the gross number and size of tumor nodules (Fig. 3b). Hence, IL-12-LNP is therapeutically effective in the treatment of transgenic mice with MYC-driven HCC.

Hematoxylin and Eosin (H\&E) staining was performed on livers from IL-12-LNP versus NST-LNP treated groups (Figs. 3c-d). Tumor tissues from mice treated with IL-12-LNP but not NST-LNP were associated with decreased number of tumor cells that were replaced with connective tissue (Fig. 3c). These findings suggest that the IL-12-LNP therapeutic can suppress HCC formation. 


\section{IL-12-LNP does not suppress MYC to cause HCC regression}

In our transgenic mouse model of MYC-driven $\mathrm{HCC}$, tumors completely regress upon MYC inactivation (Fig. 1b, Model) [25]. Hence, a trivial explanation for the decreased tumor growth in IL-12-LNP treated mice is that the therapy was reducing MYC levels. Immunohistochemistry (IHC) comparing transgenic human MYC (hMYC) protein expression in liver tumor tissues between control (NST-LNP) and IL-12-LNP-treated groups demonstrated no differences in MYC protein levels (Fig. 3d). As expected, normal liver tissues surrounding the tumor from both groups do not express the transgenic hMYC protein (Fig. 3e). Hence, IL-12-LNP does not suppress tumor growth by decreasing MYC expression.

\section{Treatment with IL-12-LNP causes HCC regression by inducing an anti-tumor immunological response}

IL-12-LNP-treated transgenic mice exhibited changes consistent with the induction of an immune response. The administration of IL-12-LNP significantly increased splenic weights of both healthy (FVB/N, Additional file 2a), and HCC-bearing mice (Additional file $2 \mathrm{~b}$ ), consistent with previously observed hematologic effects of IL-12 treatment, such as immune activation [41]. Additionally, we observed that IL-12-LNP treatment of HCC-bearing mice significantly upregulated the production of IFN $\gamma$ mRNA in the normal liver tissue surrounding the tumor when compared to NST-LNP treated controls (Additional file 2c). Increased splenic volume (Additional file $2 \mathrm{a}-\mathrm{b}$ ) and induction of IFNy mRNA (Additional file 2c) following IL-12-LNP treatment suggest that IL-12 may potentially induce anti-tumor immunological changes.

Next, using IHC, we measured the anti-tumor immunological changes induced by IL-12-LNP treatment. IL-12 has been previously shown to induce anti-tumor immune surveillance through the recruitment of host immune cells such as $\mathrm{CD}^{+} \mathrm{CD}^{+}$helper T-lymphocytes [7]. Indeed, we found that IL-12-LNP but not NST-LNP-treated mice had increased recruitment of $\mathrm{CD}^{+}$pan $\mathrm{T}$ cells both within the tumor (intratumoral immune cells, Fig. 4a), and in the surrounding normal liver tissue (Fig. 4b). Quantification of representative tumor images confirmed that there was a significant increase of $\mathrm{CD}^{+}$pan $\mathrm{T}$ cell in IL-12-LNP-treated mice (Fig. 4a). Of note, normal liver tissues surrounding the tumor in the IL-12-LNP treated group showed an accumulation of $\mathrm{CD}^{+}$pan $\mathrm{T}$ cells around blood vessels (Fig. $4 \mathrm{~b}$, right), in contrast to control mice (Fig. 4b, left), suggesting that circulating $\mathrm{T}$ cells may be recruited to the tumor site from the vasculature (Fig. 4b).

We performed further IHC-based immunological characterization of the liver tumor tissue and the surrounding normal livers of NST-LNP and IL-12-LNP-treated mice by comparing recruitment of $\mathrm{CD}^{+}$immune cells (including $\mathrm{CD}^{+} \mathrm{T}$ helper, macrophages and dendritic cells (DCs)) between the two groups (Figs. 4c-d). Similar to that observed for $\mathrm{CD}^{+}$pan $\mathrm{T}$ cells, we observed that treatment with IL-12-LNP promoted a robust recruitment of $\mathrm{CD} 4^{+}$ immune cells both into the tumor and surrounding normal liver tissue when compared to control mice treated with NST-LNP (Figs. 4c-d). Quantification of representative tumor images confirmed that there was a significant increase of $\mathrm{CD}^{+}$immune cells in IL-12-LNP-treated mice (Fig. 4c).

Our results suggested that the recruitment of a $\mathrm{CD}^{+}$ $\mathrm{CD}^{+}$helper $\mathrm{T}$ cell-mediated immune response (Figs. $4 \mathrm{a}-\mathrm{d})$ might in part account for reduced HCC growth after IL-12-LNP-treatment. Hence, we evaluated the activation status of the recruited helper T-lymphocytes post IL-12-LNP treatment by comparing the levels of CD44 (a marker of $\mathrm{T}$ cell activation that is expressed by antigen-experienced $\mathrm{T}$ cells $[42,43])$ between control NST-LNP and IL12-LNP-treated groups (Figs. 4e-f). Quantification of representative tumor images confirmed that there was a significant increase of CD44 $4^{+}$immune cells in IL-12-LNP-treated mice (Fig. 4e). IL-12 and TCR stimulation are known to enhance CD44 expression [44]. Of note, we observed an increase in the number of CD $44^{+}$immune cells recruited to tumor and surrounding normal liver tissue in IL-12-LNP treated mice in comparison to control mice (Figs. 4e-f). CD44 expression on helper $\mathrm{CD}^{+} \mathrm{CD}^{+} \mathrm{T}$ cells is known to promote Th1 responses by enhancing the production of IFN $\gamma[45$, 46], a phenomenon we also observe in our HCC model (Additional file 2c). We therefore conclude that recruitment of activated $\mathrm{CD} 44^{+} \mathrm{CD} 3^{+} \mathrm{CD} 4^{+} \mathrm{T}$ helper lymphocytes into the liver tumor and the surrounding normal liver tissue (Fig. 4) forms an integral part of the anti-HCC immunological response to IL-12-LNP treatment.

\section{Discussion}

We demonstrate that lipid nanoparticle based delivery of IL-12 mRNA therapy is effective in suppressing MYC-driven hepatocellular carcinogenesis (Fig. 5, Model). We observed both a reduction in tumor growth through dynamic measurements by MRI and an increase in overall survival in HCC-bearing mice. IL-12-LNP treatment was associated with reduced tumor burden and increased infiltration of activated (CD44. $4^{+}$Figs. $\left.4 \mathrm{e}-\mathrm{f}\right)$ immune cells such as helper $\mathrm{CD}^{+}{ }^{+} \mathrm{CD} 4^{+} \mathrm{T}$ cells into the tumor (Figs. 4a-d). Hence, we have obtained preclinical results suggesting that IL-12-LNP may be an effective immunotherapy against human HCC.

Our study expands upon previous reports suggesting that IL-12 therapy may be an effective treatment for cancer. First, in contrast to previously designed IL-12 
a Liver tumor at the time of mouse sacrifice $\left(C D 3^{+}\right.$immune cells $)$
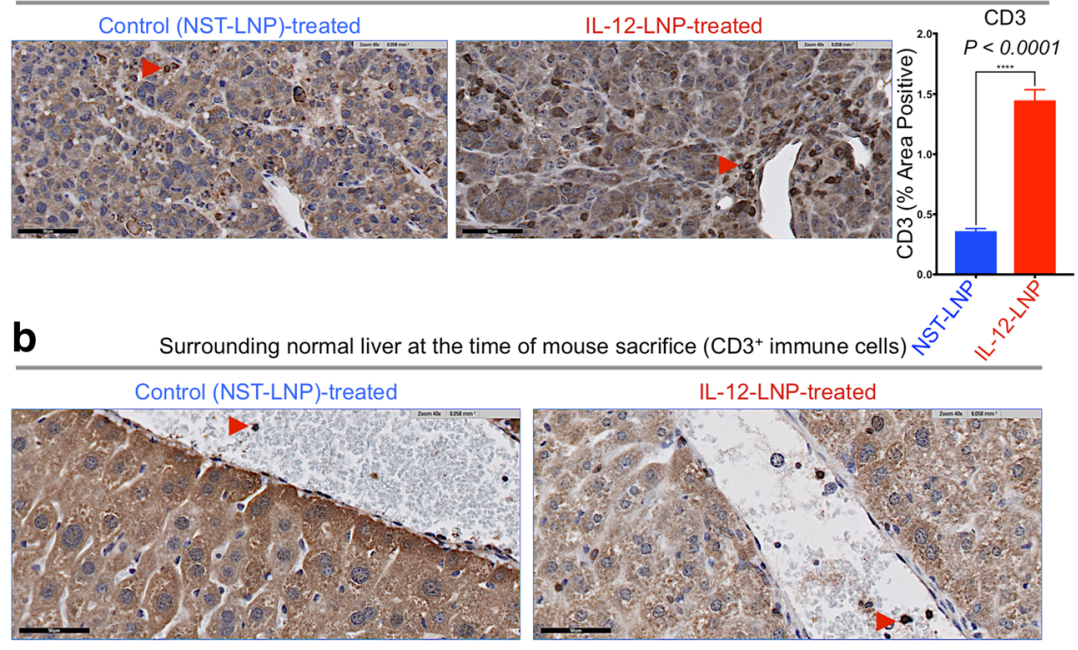

C Liver tumor at the time of mouse sacrifice $\left(C D 4^{+}\right.$immune cells)
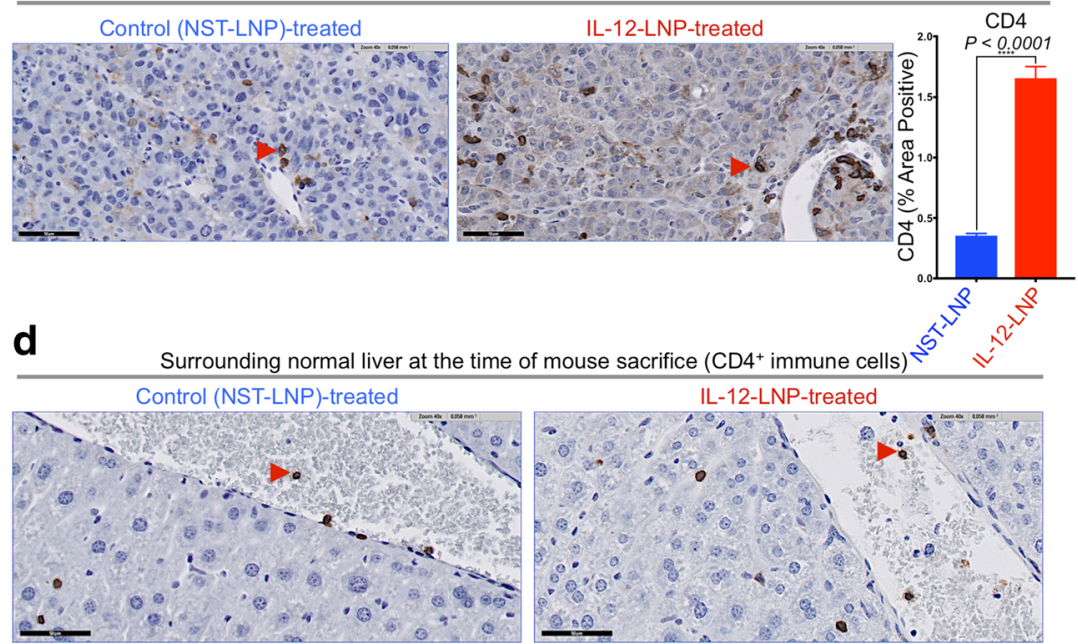

e Liver tumor at the time of mouse sacrifice (CD44+ immune cells)

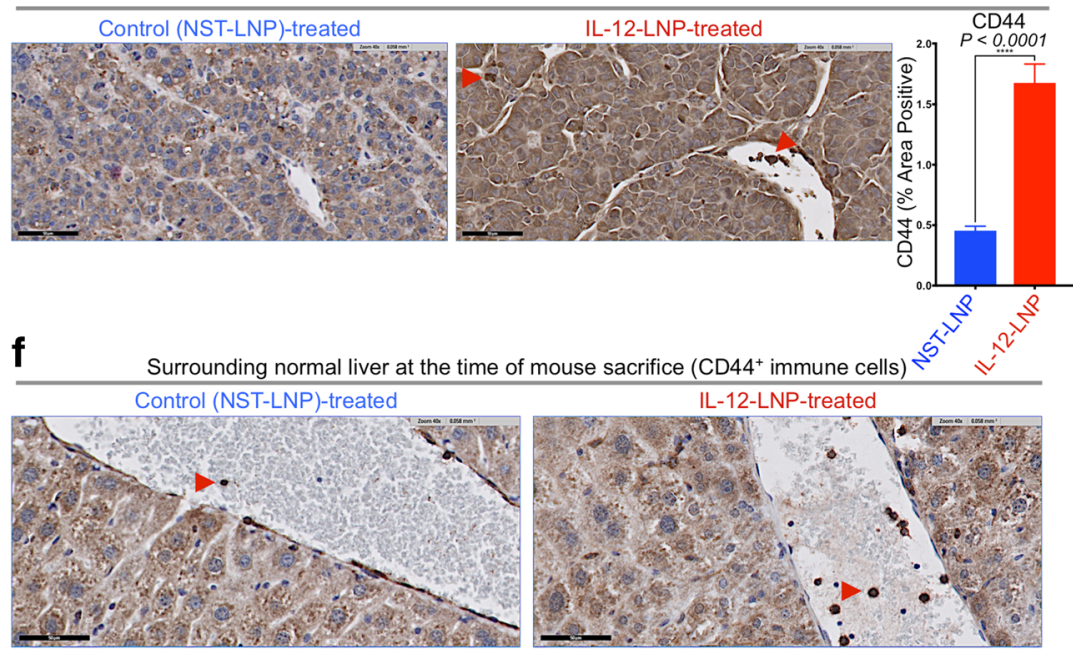

Fig. 4 (See legend on next page.) 
(See figure on previous page.)

Fig. 4 HCC clearance by IL-12-LNP is associated with the recruitment of activated immune cells. a-b Immunohistochemistry for CD3+ pan T cells in liver tumor (a), and surrounding normal liver (b) tissues isolated from control (NST-LNP, $n=6$ ), and IL-12 treated (IL-12-LNP, $n=5)$ MYC-driven HCC mice. c-d Immunohistochemistry for CD4+ immune cells (helper T, macrophages and DCs) in the liver tumor (c), and surrounding normal liver (d) tissues isolated from control (NST-LNP, $n=6$ ), and IL-12 treated (IL-12-LNP, $n=5$ ) MYC-driven HCC mice. e-f Immunohistochemistry for activated CD44+ T cells in liver tumor (e), and surrounding normal liver (f) tissues isolated from control (NST-LNP, $n=6$ ), and IL-12 treated (IL-12LNP, $n=5)$ MYC-driven HCC mice. Quantification for percent area positive of intratumoral CD3+ (a), CD4+ (c), and CD44+ (e) immune cells is provided to the right of the corresponding $I H C$ images. Quantification is an average of 5 representative IHC images for each group. Red arrowheads within IHC images indicate positive staining for the specific types of immune cells measured. One representative image is shown from each group of mice. Scale bars $=50 \mu \mathrm{m}$. P-values were calculated using Student's t-test. P values: ${ }^{* * * *} \mathrm{p}<0.0001$

therapies, the liposomal IL-12 messenger mRNA therapeutic (IL-12-LNP) does not cause general cytotoxic effects (Figs. 1d-e, Additional file 1a), that have been observed by others $[47,48]$. Second, the IL-12-LNP was effectively delivered to the HCC (Fig. 1c) achieving what appears to be associated with a more durable anti-tumor response. Third, we are the first to show a reduction in HCC tumor growth upon IL-12 therapy, by using dynamic MRI measurements (Figs. 2a-e).

Many studies have demonstrated that IL-12 therapy against tumors is associated with the recruitment of an immune response [47, 49-51]. We found that IL-12-LNP did not alter MYC protein levels (Fig. 3d), but did result in a robust recruitment of activated $\left(\mathrm{CD} 44^{+}\right.$, Figs. 4e-f) helper T-lymphocytes $\left(\mathrm{CD}^{+} \mathrm{CD}^{+}\right.$, Figs. $\left.4 \mathrm{a}-\mathrm{d}\right)$, and other $\mathrm{CD}^{+}{ }^{+}$immune cells (Figs. 4c-d). A recent study demonstrated that IL-12 promotes HCC regression by polarizing the macrophages to the M1 phenotype [18]. Further studies need to be conducted to evaluate the phenotype and activation status of $\mathrm{CD} 4^{+}$immune cells other than T cells, such as macrophages and dendritic cells (DCs).

Finally, many investigators are attempting to identify drugs that target MYC for the treatment of cancer. However, we have suggested recently, that MYC-driven

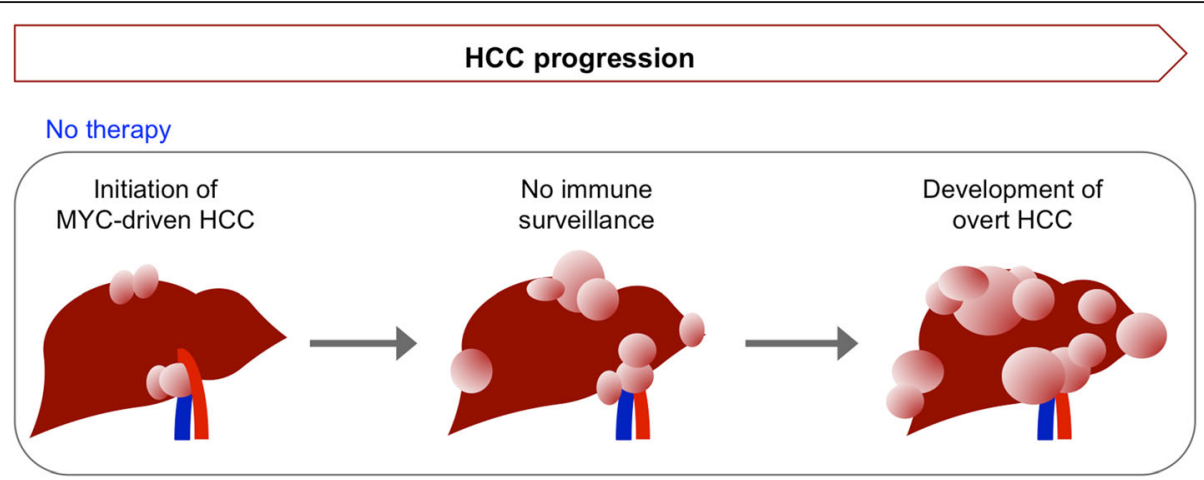

IL-12 mRNA-LNP therapy

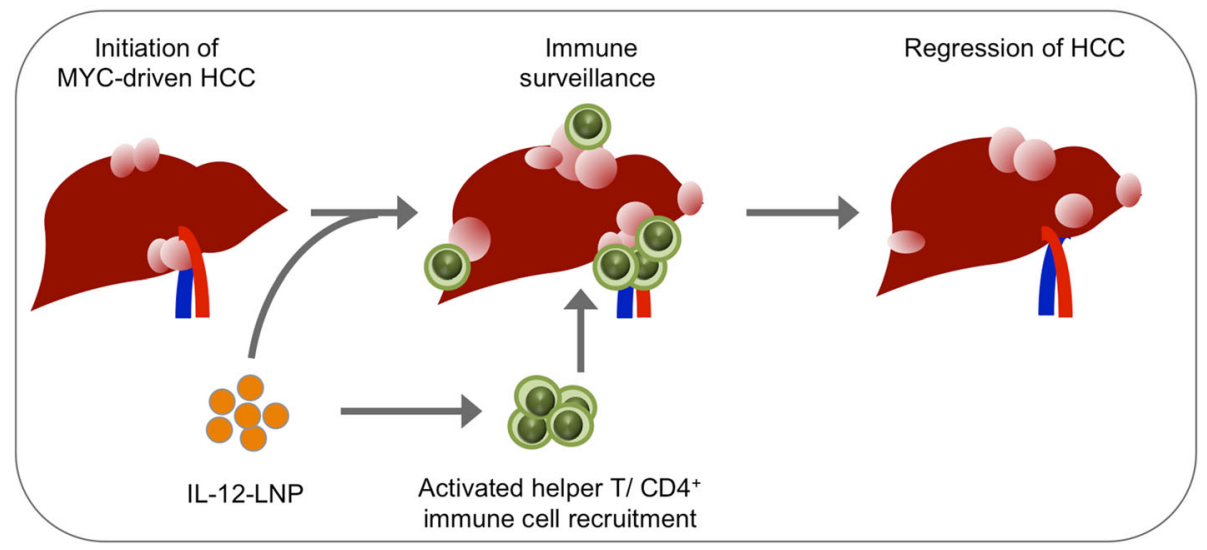

Fig. 5 Proposed model by which IL-12-LNP impedes HCC tumor progression. Model depicting the process of HCC progression in the absence (no therapy), and after administration of IL-12-LNP therapy. Intravenous administration of LNPs containing IL-12 mRNA ensures delivery directly to the liver tumor, where it recruits CD44+ CD3+ CD4+ helper T cells and other CD4+ immune cells from the general circulation. CD4+ immune cells then cause HCC regression by raising an immune response to the tumor, and block progression to overt HCC 
cancers may be generally sensitive to immune therapies, since MYC appears to play a major role in suppressing the immune response against tumors [52, 53]. Moreover, therapies that target the vulnerability of MYC-driven cancers rather than its direct suppression may avoid the toxicity that would be expected in normal tissues. Our current study demonstrates that IL-12 may be one such therapy, which if designed robustly can be delivered specifically to the tumor and can block tumor growth without damaging surrounding normal cells. Our work should inspire further efforts to develop IL-12-LNP as a therapeutic for human cancer.

\section{Additional files}

Additional file 1: IL-12-LNP does not induce toxicity in healthy mice. (a) Evaluation of toxicity of IL-12 oligonucleotide therapy (IL-12-LNP) as measured by change in body weight of healthy mice post treatment as compared to NST-LNP-treated healthy mice ( $n=3$ mice per group). (TIFF $14745 \mathrm{~kb}$ )

Additional file 2: HCC clearance by IL-12-LNP is associated with anti-tumor immunological changes. (a-b) Splenic weights depicted as percentage of initial body weights for NST-LNP controls $(n=7)$, and IL-12-LNP-treated $(n=7)$ healthy mice (a); and for NST-LNP controls $(n=7)$, and IL-12-LNP-treated $(n=7)$ MYC-driven HCC mice (b). Data are represented as mean \pm s.d. (c) Quantitative real time PCR comparing mRNA levels of IFNy between NST-LNP $(n=7)$ and IL-12-LNP $(n=7)$ treated HCC bearing mice. Each dot represents a single mouse and is the average of three technical replicates. Data are represented as median \pm interquartile range. (TIFF $14745 \mathrm{~kb}$ )

\section{Abbreviations}

HCC: Hepatocellular Carcinoma; GEO: Gene Expression Omnibus; MRI: Magnetic Resonance Imaging; IL-12: Interleukin-12; MYC: v-myc avian myelocytomatosis viral oncogene homolog; OS: overall survival; IHC: immunohistochemistry; NK cell: natural killer cell; TCR: T cell receptor; IL12R: Interleukin-12 receptor; LNP: Lipid Nanoparticle; NST: no start codon; hMYC: human MYC; mRNA: messenger RNA; GOT1: Glutamic-Oxaloacetic Transaminase 1; ALPL: Alkaline Phosphatase; GPT: Glutamic Pyruvic Transaminase; DCs: Dendritic Cells

\section{Acknowledgements}

We thank M. Gaarnas, Y. Zhou, and J. Frederick from Onkaido for providing the LNPs. We thank L. Pisani in the Department of Radiology Stanford University for providing MRI support. We thank P. Chu in the Department of Pathology at Stanford University for carrying out tissue sectioning for immunohistochemical analyses.

\section{Funding}

This work is supported by the following grants: $\mathrm{NIH} / \mathrm{NCl}$ CA170378PQ2, 1U01CA188383-01/S1, S10RR026917-01, Emerson Collective Cancer Research Fund, and funding from Moderna (Onkaido Therapeutics) to D. Felsher.

\section{Availability of data and materials}

Onkaido Therapeutics provided NST-LNP control and the IL-12-LNP mRNA therapy.

\section{Authors' contributions}

DWF conceived the study. IL and SS developed the experimental methodology. IL conducted majority of the experiments. SS wrote the manuscript. VB helped with performing and analyzing $\mathrm{IHC}$ experiments. AM helped with intravenous injections of IL-12-LNP and the NST-LNP controls. RD provided expert scientific advice on HCC pathogenesis. AM, VB, and MG provided helpful scientific input on the manuscript. IL and DWF edited the manuscript, and DWF provided administrative, technical, and material support. DWF supervised the study. All authors read and approved the final manuscript.

\section{Ethics approval and consent to participate}

All procedures with animals were carried out under the guidelines of the National Institutes of Health and in accordance with approved Stanford University APLAC animal protocol (10563).

\section{Consent for publication}

All authors of the manuscript have read and agreed to its content and are accountable for all aspects of the accuracy and integrity of the manuscript. The manuscript is original, has not already been published, and is not currently under consideration by another journal.

\section{Competing interests}

The authors declare that they have no competing interests.

\section{Publisher's Note}

Springer Nature remains neutral with regard to jurisdictional claims in published maps and institutional affiliations.

\section{Author details}

'Division of Medical Oncology, Departments of Medicine and Pathology, Stanford University, CA, Stanford, USA. ${ }^{2}$ Division of Gastroenterology and Hepatology, Stanford University, Stanford, CA, USA.

Received: 3 July 2018 Accepted: 18 October 2018

Published online: 20 November 2018

\section{References}

1. Colombo MP, Trinchieri G. Interleukin-12 in anti-tumor immunity and immunotherapy. Cytokine Growth Factor Rev. 2002;13:155-68.

2. Noguchi $Y$, Jungbluth $A$, Richards EC, Old LJ. Effect of interleukin 12 on tumor induction by 3-methylcholanthrene. Proc Natl Acad Sci U S A. 1996; 93:11798-801.

3. Vizler C, Rosato A, Calderazzo F, Quintieri L, Fruscella P, Wainstok de Calmanovici $R$, et al. Therapeutic effect of interleukin 12 on mouse haemangiosarcomas is not associated with an increased anti-tumour cytotoxic T-lymphocyte activity. Br J Cancer. 1998;77:656-62.

4. Boggio K, Di Carlo E, Rovero S, Cavallo F, Quaglino E, Lollini PL, et al. Ability of systemic interleukin-12 to hamper progressive stages of mammary carcinogenesis in HER2/neu transgenic mice. Cancer Res. 2000;60:359-64.

5. Brunda MJ, Luistro L, Warrier RR, Wright RB, Hubbard BR, Murphy M, et al. Antitumor and antimetastatic activity of interleukin 12 against murine tumors. J Exp Med. 1993;178:1223-30.

6. Kishima H, Shimizu K, Miyao Y, Mabuchi E, Tamura K, Tamura M, et al. Systemic interleukin 12 displays anti-tumour activity in the mouse central nervous system. Br J Cancer. 1998;78:446-53.

7. Trinchieri G. Interleukin-12: a proinflammatory cytokine with immunoregulatory functions that bridge innate resistance and antigenspecific adaptive immunity. Annu Rev Immunol. 1995:13:251-76.

8. Kobayashi M, Fitz L, Ryan M, Hewick RM, Clark SC, Chan S, et al. Identification and purification of natural killer cell stimulatory factor (NKSF), a cytokine with multiple biologic effects on human lymphocytes. J Exp Med. 1989:170:827-45.

9. Presky $\mathrm{DH}$, Yang $\mathrm{H}$, Minetti $\amalg$, Chua AO, Nabavi N, Wu CY, et al. A functional interleukin 12 receptor complex is composed of two beta-type cytokine receptor subunits. Proc Natl Acad Sci U S A. 1996;93:14002-7.

10. Grohmann U, Belladonna ML, Bianchi R, Orabona C, Ayroldi E, Fioretti MC, et al. IL-12 acts directly on DC to promote nuclear localization of NF-kappaB and primes DC for IL-12 production. Immunity. 1998;9:315-23.

11. Airoldi I, Gri G, Marshall JD, Corcione A, Facchetti P, Guglielmino R, et al. Expression and function of $\mathrm{IL}-12$ and $\mathrm{IL}-18$ receptors on human tonsillar $\mathrm{B}$ cells. J Immunol. 2000;165:6880-8.

12. Afkarian M, Sedy JR, Yang J, Jacobson NG, Cereb N, Yang SY, et al. T-bet is a STAT1-induced regulator of IL-12R expression in naïve CD4+ T cells. Nat Immunol. 2002;3:549-57.

13. Szabo SJ, Dighe AS, Gubler U, Murphy KM. Regulation of the interleukin (IL)12R beta 2 subunit expression in developing T helper 1 (Th1) and Th2 cells. J Exp Med. 1997;185:817-24.

14. Salem ML, Kadima AN, Zhou Y, Nguyen CL, Rubinstein MP, Demcheva M, et al. Paracrine release of IL-12 stimulates IFN-gamma production and dramatically enhances the antigen-specific $T$ cell response after vaccination with a novel peptide-based cancer vaccine. J Immunol. 2004;172:5159-67. 
15. Parker BS, Rautela J, Hertzog PJ. Antitumour actions of interferons: implications for cancer therapy. Nat Rev Cancer. 2016;16:131-44.

16. Cavallo F, Signorelli P, Giovarelli M, Musiani P, Modesti A, Brunda MJ, et al. Antitumor efficacy of adenocarcinoma cells engineered to produce interleukin 12 (IL-12) or other cytokines compared with exogenous IL-12. J Natl Cancer Inst. 1997:89:1049-58.

17. Harada N, Shimada M, Okano S, Suehiro T, Soejima Y, Tomita Y, et al. IL-12 gene therapy is an effective therapeutic strategy for hepatocellular carcinoma in immunosuppressed mice. J Immunol. 2004;173:6635-44

18. Wang Q, Cheng F, Ma T-T, Xiong H-Y, Li Z-W, Xie C-L, et al. Interleukin-12 inhibits the hepatocellular carcinoma growth by inducing macrophage polarization to the M1-like phenotype through downregulation of Stat-3. Mol Cell Biochem. 2016;415:157-68.

19. Zilocchi C, Stoppacciaro A, Chiodoni C, Parenza M, Terrazzini N, Colombo MP. Interferon gamma-independent rejection of interleukin 12-transduced carcinoma cells requires CD4+ T cells and granulocyte/macrophage colonystimulating factor. J Exp Med. 1998;188:133-43.

20. Tugues S, Burkhard SH, Ohs I, Vrohlings M, Nussbaum K, vom Berg J, et al. New insights into IL-12-mediated tumor suppression. Cell Death Differ. 2015;22:237-46

21. White DL, Thrift AP, Kanwal F, Davila J, El-Serag HB. Incidence of Hepatocellular Carcinoma in All 50 United States, From 2000 Through 2012. Gastroenterology. 2017;152:812-20.e5.

22. Llovet JM, Ricci S, Mazzaferro V, Hilgard P, Gane E, Blanc J-F, et al. Sorafenib in advanced hepatocellular carcinoma. N Engl J Med. 2008;359:378-90.

23. Bruix J, Qin S, Merle P, Granito A, Huang Y-H, Bodoky G, et al. Regorafenib for patients with hepatocellular carcinoma who progressed on sorafenib treatment (RESORCE): a randomised, double-blind, placebo-controlled, phase 3 trial. Lancet Lond Engl. 2017;389:56-66.

24. Llovet JM, Hernandez-Gea V. Hepatocellular carcinoma: reasons for phase III failure and novel perspectives on trial design. Clin Cancer Res Off J Am Assoc Cancer Res. 2014;20:2072-9.

25. Beer S, Zetterberg A, Ihrie RA, McTaggart RA, Yang Q, Bradon N, et al. Developmental context determines latency of MYC-induced tumorigenesis. PLoS Biol. 2004;2:e332.

26. Chan K-L, Guan X-Y, Ng IO-L. High-throughput tissue microarray analysis of c-myc activation in chronic liver diseases and hepatocellular carcinoma. Hum Pathol. 2004:35:1324-31.

27. Yuen MF, Wu PC, Lai VC, Lau JY, Lai CL. Expression of C-Myc, C-Fos, and Cjun in hepatocellular carcinoma. Cancer. 2001;91:106-12.

28. Kawate S, Fukusato T, Ohwada S, Watanuki A, Morishita Y. Amplification of c-myc in hepatocellular carcinoma: correlation with clinicopathologic features, proliferative activity and p53 overexpression. Oncology. 1999; 57:157-63.

29. Peng SY, Lai PL, Hsu HC. Amplification of the c-myc gene in human hepatocellular carcinoma: biologic significance. J Formos Med Assoc Taiwan Yi Zhi. 1993;92:866-70.

30. Shachaf CM, Kopelman AM, Arvanitis C, Karlsson A, Beer S, Mandl S, et al. MYC inactivation uncovers pluripotent differentiation and tumour dormancy in hepatocellular cancer. Nature. 2004;431:1112-7.

31. Simile MM, De Miglio MR, Muroni MR, Frau M, Asara G, Serra S, et al. Downregulation of c-myc and cyclin D1 genes by antisense oligodeoxy nucleotides inhibits the expression of E2F1 and in vitro growth of HepG2 and Morris 5123 liver cancer cells. Carcinogenesis. 2004;25:333-41.

32. Yin X, Giap C, Lazo JS, Prochownik EV. Low molecular weight inhibitors of Myc-max interaction and function. Oncogene. 2003;22:6151-9.

33. Lin C-P, Liu J-D, Chow J-M, Liu C-R, Liu HE. Small-molecule c-Myc inhibitor 10058-F4, inhibits proliferation, downregulates human telomerase reverse transcriptase and enhances chemosensitivity in human hepatocellular carcinoma cells. Anti-Cancer Drugs. 2007;18:161-70.

34. Hoshida Y, Villanueva A, Kobayashi M, Peix J, Chiang DY, Camargo A, et al. Gene expression in fixed tissues and outcome in hepatocellular carcinoma. N Engl J Med. 2008;359:1995-2004.

35. Dhanasekaran R, Gabay-Ryan M, Baylot V, Lai I, Mosley A, Huang X, et al. Anti-miR17 Therapy Delays Tumorigenesis in MYC-driven Hepatocellular Carcinoma (HCC). Oncotarget. 2017;5. https://doi.org/10.18632/oncotarget.22342.

36. Kormann MSD, Hasenpusch G, Aneja MK, Nica G, Flemmer AW, HerberJonat $\mathrm{S}$, et al. Expression of therapeutic proteins after delivery of chemically modified mRNA in mice. Nat Biotechnol. 2011;29:nbt.1733.
37. Mclvor RS. Therapeutic delivery of mRNA: the medium is the message., Therapeutic Delivery of mRNA: The Medium Is the Message. Mol Ther J Am Soc Gene Ther Mol Ther. 2011;19:822, 822-3.

38. Karikó K, Muramatsu H, Ludwig J, Weissman D. Generating the optimal mRNA for therapy: HPLC purification eliminates immune activation and improves translation of nucleoside-modified, protein-encoding mRNA. Nucleic Acids Res. 2011;39:e142.

39. Fotin-Mleczek M, Duchardt KM, Lorenz C, Pfeiffer R, Ojkić-Zrna S, Probst J, et al. Messenger RNA-based vaccines with dual activity induce balanced TLR-7 dependent adaptive immune responses and provide antitumor activity. J Immunother. 2011;34:1-15.

40. Hoerr I, Obst R, Rammensee HG, Jung G. In vivo application of RNA leads to induction of specific cytotoxic T lymphocytes and antibodies. Eur J Immunol. 2000;30:1-7.

41. Sarmiento UM, Riley JH, Knaack PA, Lipman JM, Becker JM, Gately MK, et al. Biologic effects of recombinant human interleukin-12 in squirrel monkeys (Sciureus saimiri). Lab Investig J Tech Methods Pathol. 1994;71:862-73.

42. Budd RC, Cerottini JC, Horvath C, Bron C, Pedrazzini T, Howe RC, et al. Distinction of virgin and memory T lymphocytes. Stable acquisition of the Pgp-1 glycoprotein concomitant with antigenic stimulation. J Immunol. 1987;138:3120-9.

43. Föger N, Marhaba R, Zöller M. CD44 supports T cell proliferation and apoptosis by apposition of protein kinases. Eur J Immunol. 2000;30:2888-99.

44. Blass SL, Puré E, Hunter CA. A role for CD44 in the production of IFN- $\gamma$ and immunopathology during infection with toxoplasma gondii. J Immunol. 2001;166:5726-32

45. Guan H, Nagarkatti PS, Nagarkatti M. Role of CD44 in the differentiation of Th1 and Th2 cells: CD44-deficiency enhances the development of Th2 effectors in response to SRBC and chicken ovalbumin. J Immunol. 2009;183:172-80.

46. Schumann J, Stanko K, Schliesser U, Appelt C, Sawitzki B. Differences in CD44 surface expression levels and function discriminates IL-17 and IFN- $\gamma$ producing helper T cells. PLoS One. 2015;10. https://doi.org/10.1371/journal. pone.0132479.

47. Lasek W, Zagożdżon R, Jakobisiak M. Interleukin 12: still a promising candidate for tumor immunotherapy? Cancer Immunol Immunother. 2014; 63:419-35.

48. Gately MK, Gubler U, Brunda MJ, Nadeau RR, Anderson TD, Lipman JM, et al. Interleukin-12: a cytokine with therapeutic potential in oncology and infectious diseases. Ther Immunol. 1994;1:187-96.

49. Zeh HJ, Hurd S, Storkus WJ, Lotze MT. Interleukin-12 promotes the proliferation and cytolytic maturation of immune effectors: implications for the immunotherapy of cancer. J Immunother Emphas Tumor Immunol Off Soc Biol Ther. 1993;14:155-61.

50. Otani T, Nakamura S, Toki M, Motoda R, Kurimoto M, Orita K. Identification of IFN-gamma-producing cells in IL-12/IL-18-treated mice. Cell Immunol. 1999:198:111-9.

51. Trinchieri G, Wysocka M, D'Andrea A, Rengaraju M, Aste-Amezaga M, Kubin M, et al. Natural killer cell stimulatory factor (NKSF) or interleukin-12 is a key regulator of immune response and inflammation. Prog Growth Factor Res. 1992:4:355-68.

52. Rakhra K, Bachireddy P, Zabuawala T, Zeiser R, Xu L, Kopelman A, et al. CD4(+) T cells contribute to the remodeling of the microenvironment required for sustained tumor regression upon oncogene inactivation. Cancer Cell. 2010;18:485-98.

53. Casey SC, Tong L, Li Y, Do R, Walz S, Fitzgerald KN, et al. MYC regulates the antitumor immune response through CD47 and PD-L1. Science. 2016;352:227-31.

Ready to submit your research? Choose BMC and benefit from:

- fast, convenient online submission

- thorough peer review by experienced researchers in your field

- rapid publication on acceptance

- support for research data, including large and complex data types

- gold Open Access which fosters wider collaboration and increased citations

- maximum visibility for your research: over $100 \mathrm{M}$ website views per year

At $\mathrm{BMC}$, research is always in progress.

Learn more biomedcentral.com/submissions 